\title{
PARTicipación estudiantil COMUnitaria
}

\author{
Community student participation
}

\section{Silvia del Rosario López de Maturana Luna Universidad de La Serena (ULS), Chile}

RESUMEN: La formación en comunidad de las alumnas de Pedagogía en Educación Parvularia de la Universidad de La Serena, Chile, implica una participación permanente en los procesos socioculturales que ocurren en sus prácticas, lo que disminuye el riesgo de vivir experiencias escolarizantes y acomodaticias en su formación profesional. Ellas estudian de manera no lineal, tensando el orden programático de la clase con la emergencia del devenir contingente de la comunidad urbano-marginal en la que participan, proceso del cual darán cuenta a lo largo de sus cuatro años de formación universitaria. A medida que las alumnas van descubriendo la comunidad van reconociendo las sinergias educativas que emergen en los espacios y tiempos informales donde se desarrolla la vida comunitaria, y generan una recursividad de transformación de los procesos de enseñanza y de aprendizaje universitarios.

Palabras ClaVe: pedagogía comunitaria; participación; procesos socioculturales.

RESUM: La formació en comunitat de les alumnes de Pedagogia en Educació Parvulària de la Universitat de la Serena, Xile, implica una participació permanent en els processos socioculturals que tenen lloc en les seues pràctiques, la qual cosa minva el risc de viure experiències escolaritzants i acomodatícies en la seua formació professional. Elles estudien de manera no lineal i tensen l'ordre programàtic de la classe amb l'emergència de l'esdevenir contingent de la comunitat urbanomarginal en què participen, procés del qual donaran compte al llarg dels seus quatre anys de formació universitària. A mesura que les alumnes descobreixen la comunitat, reconeixen les sinergies educatives que emergeixen en els espais i temps informals on es desenvolupa la vida 
comunitària. Generen, així, una recursivitat de transformació dels processos d'ensenyament i d'aprenentatge universitaris.

Paraules ClaU: pedagogia comunitària, participació, processos socioculturals.

ABSTRACT: Community training for students of pedagogy in early childhood education at the University of La Serena, Chile, involves permanent participation in the sociocultural processes that arise in their practical sessions. This participation reduces the risk of schooling and accommodative experiences in their vocational training. The students' learning process is non-linear, seeking tensions between the programmed order of the class with the emergence of a possible transformation in the marginal urban community in which they participate, a process they will recount throughout their four-year university education. As the students discover the community, they recognize the educational synergies that emerge in the informal spaces and times where community life takes place, generating resources for the transformation of university teaching and learning processes.

KEYWORDS: community pedagogy; participation; sociocultural processes.

\section{Participación y pedagogía comunitaria}

$\mathrm{L}$ a formación en comunidad ${ }^{1}$ del alumnado universitario implica la participación permanente en los procesos socioculturales en que se dan sus prácticas, lo que permite comprender el sentido de las teorías, vínculos, redes, y soportes sociales que se crean en la relación entre los contextos informales, que se dan fuera de las aulas, y los formales, que se generan dentro de ellas. Introducirse en las comunidades conlleva mirar desde otro prisma a las personas que transitan por las calles, cada una con sus peculiares características y reconocer a las familias, niños, jóvenes, adultos y ancianos que forman parte

1. Se entiende por comunidad un sector urbano marginal de la ciudad. 
de lo cotidiano. Ello favorece la generación de experiencias transformadoras y disminuye el riesgo de las rutinas escolarizantes ${ }^{2}$ (Calvo, 2016), acomodaticias y complacientes que, por lo general, caracterizan las aulas en los diferentes niveles de formación.

La participación trasciende el mero hecho de asistir a una reunión, visitar la comunidad en grupo, o apoyar una idea, puesto que conlleva comprender en profundidad y formar parte activa de lo que ocurre en las comunidades, los procesos históricos que las han construido, las condiciones de alienación y atomización que someten a sus miembros, y también «las condiciones óptimas de organización, conocimiento e instrumentación técnica que les permitan actuar efectivamente en la transformación de su realidad» (Arango, 2008, p. 165). Participar en experiencias conjuntas con los miembros de la comunidad en exposiciones artísticas, obras de teatro, cine arte, literatura y otro tipo de expresiones son experiencias vitales que permiten al alumnado conocer la realidad en la que viven los niños y niñas a los que les tocará educar, y contribuyen a su formación profesional más que ninguna asignatura de la malla curricular, puesto que les entrega sólidos valores sobre los que construir sus saberes, al tiempo que les permite trascender la mirada paternalista con la que, generalmente, se ve o define a las comunidades urbano-marginales o rurales. Generalmente, las comunidades más pobres son definidas hegemónicamente, desde visiones funcionalistas y estereotipadas, como un grupo de individuos que necesitan de ayuda, porque carecen de recursos propios que les permitan progresar. Ello hace necesario replantear una pedagogía contrahegemónica que libere el pensamiento crítico del alumnado para que sean capaces de cuestionar el orden preestablecido, donde lo comunitario no se entienda como un espacio físico, sino como «la manifestación viva de la comunidad, pues es desde lo comunitario que se construye el proyecto de futuro» (Flores, 2014, p. 76).

Congruente con lo señalado, la carrera de Pedagogía en Educación Parvularia de la Universidad de La Serena, desde el año 2012, propuso e instauró, dentro del plan de estudios, la Pedagogía Comunitaria y replanteó el currí-

2. Distinguimos entre educación y escolarización, la primera es la creación de relaciones posibles y la segunda, la repetición de patrones preestablecidos (Calvo, 2016). 
culum de formación, para llevar a las alumnas ${ }^{3}$ a descubrir y a reconocerse como sujetos en la comunidad, a comprender la vida de los actores sociales, conocer sus sufrimientos, alegrías, aspiraciones y luchas, porque la formación pedagógica no es una tarea simplemente teórica que ocurre dentro de las instituciones formadoras, sino fundamentalmente práctica y llevada a cabo en los contextos donde ocurre la vida misma (Martín-Baró, 2006). En conjunto con el profesorado de la carrera, se instaló el sello comunitario en la formación como eje transversal que permea el currículum ${ }^{4}$, y se consideró un imperativo educativo, social, ético y político situar a las alumnas en el entorno real donde transcurre la vida cotidiana de gran parte de los párvulos a quienes van a educar. Se pensó en reivindicar la condición humana y liberar a las alumnas del academicismo que las aliena y aleja del real sentido de la praxis transformadora. Gracias a eso, se involucraron con gusto, amor y compromiso en la tarea y en la relación con los otros en un proceso de concientización, liberación y transformación para recuperar el sentido de los espacios y tiempos comunitarios (Freire, 1993; Flores, 2014).

Es precisamente este tipo de práctica comunitaria la que permite a las alumnas valorar a cada miembro de la comunidad como poseedor de experiencias y conocimientos válidos que los legitiman como sujetos y no como objetos medibles a través de estándares que terminan por anularlos. Eso favorece el compromiso y las buenas relaciones con los vecinos de los barrios a los que asisten, ya que contribuye a afianzar los soportes personales y sociales necesarios para la armonía psicosocial, desarrollo y bienestar de cada participante, y constituye uno de los principales dispositivos para que las personas se sientan útiles y necesarias a la sociedad, al tiempo que tienen mayores posibilidades de eliminar estructuras reproductivas de inequidad (Caron, 1996). Eso sucede en un marco de investigación acción participativa (Fals-Borda y Anisur, 1991), pertinente para los propósitos de la formación comunitaria,

3. Hasta la fecha son solamente mujeres.

4. Este proyecto fue gestado por los profesores de la carrera de Pedagogía en Educación Parvularia de la Universidad de La Serena (uls), Chile: Dr. Carlos Calvo, Dra. Yanola González y Dra. Silvia López de Maturana. Actualmente, 2016, forma parte de un proyecto de investigación de la Dirección de Investigación de la ULS, donde estamos realizando la sistematización de las experiencias de pedagogía comunitaria. Participan los profesores doctores de la carrera: Silvia López de Maturana, Yanola González, Carlos Calvo, Rossana Godoy y Clara Tirado. 
sobre todo porque no tiene un enfoque único, ya que depende de los contextos y de los momentos históricos de cada comunidad. Eso permitió trabajar en equipo para contextualizar y recopilar información básica de la inmersión de las alumnas en la comunidad; analizar críticamente los problemas considerados prioritarios; identificar y describir emergencias, nodos y sinergias que se generan gracias a la tensión entre teoría y práctica; atribuir significado educativo a la relación entre aprendizaje formal (asignaturas) e informal (práctica comunitaria); y reconocer las redes sociocomunitarias de encuadre positivo ${ }^{5}$.

Desde el primer año de ingreso, las alumnas eligen una calle en un sector urbano marginal de la ciudad donde, una vez a la semana, durante los cuatro años de su carrera, se involucran con la comunidad para trabajar pedagógicamente los barrios, las calles y las plazas del entorno. Desde el primer año levantan problemáticas desde la comunidad y las llevan a las aulas para analizarlas junto a sus profesores y profesoras de las distintas disciplinas, por ejemplo, un problema de salud detectado en el barrio puede ser analizado transversalmente en la clase de biología, sociología, psicología, teoría de la educación, entre otras. Eso no significa que se excluye el currículum formal de la carrera, sino que se enriquece y se complementa con la formación en comunidad que reciben las alumnas desde sus propias vivencias en los barrios donde participan. En un comienzo, las alumnas parten con miedos e incertidumbres puesto que, intencionalmente, no se les entregan guías de apoyo para integrarse en la comunidad, ya que deben ir aprendiéndolo durante el proceso de inmersión que dura el primer año de carrera. Existe confusión, rechazo, dudas de sus capacidades, y sensación de encontrarse perdidas, sin saber qué hacer:

«Aun no sé muy bien de qué se trata la comunidad.»

«Tampoco sabía bien lo que iba decir a la gente, sabía que tenía que saludarla y presentarme, pero después ¿qué seguía?»

«QQué puedo hablar con la gente de la comunidad para que se le haga interesante?»

5. Entendemos el encuadre positivo como el reconocimiento de las fortalezas más que de las debilidades de una comunidad. 
«Debo admitir que en ese momento no estaba entusiasmada con el proyecto, porque sentía que nadie me abriría la puerta o que solo recibiría negativas de las personas que vivian alli.»

«Aunque, la verdad, estoy un poco asustada porque no sé muy bien qué decir o cómo presentarme cuando me abran la puerta; pero bueno, tendré que dejar de lado mi nerviosismo y timidez que me caracteriza.»

«Hasta ese momento estaba bastante frustrada y tenia ganas de irme pero decidí intentar en una casa más.»

«La primera vez que nos dirigimos a la comunidad iba llena de miedos e incertidumbres, ya que llegué a un lugar totalmente desconocido para mí».

La intención de la pedagogía comunitaria es recuperar la propensión a aprender de todo ser humano que se pierde no por la maduración biológica, psicológica, social o cultural, sino por la influencia negativa de un modelo paradigmático de concebir el aprendizaje. Se espera recuperar el aprendizaje que surge desde la sorpresa, extrañeza y atracción por lo imprevisto y desafiante. Esa cualidad inherente al ser humano no sigue un orden regular ni previsible, sino aleatorio e imprevisto que tiende a autoorganizarse gracias a los patrones informales que se ordenan en torno al asombro y a los atractores antes señalados. Como muestra de que aquello se ha anquilosado, las alumnas en su primer acercamiento comunitario se cuestionan el por qué asistir a la comunidad y se ciegan a la apertura de otras posibilidades y a entender el sentido de esas prácticas; aluden a su derecho de estudiar dentro de las aulas, probablemente por la fuerza hegemónica del paradigma dominante escolar que las condujo a la mecanización y repetición de aprendizajes desvinculados del mundo circundante:

«Me gustaría encontrarle un sentido a esto, porque cada día trato de encontrarlo pero aun no aparece.»

«Vuelvo a caminar por mi calle, y trato de ver en qué forma puedo influir yo en este lugar.» 
El segundo año es de acercamiento y confianza con los vecinos, el tercero, de establecimiento de acciones comunes que denotan experiencias transformadoras, y el cuarto año es de consolidación de un proyecto sustentable, creado con los vecinos. Estas experiencias no solo transforman las comunidades, sino que son altamente transformadoras en la vida personal y formativa de las alumnas, donde cuestionan lo que significa educar a los párvulos, conocen y comprenden el entorno en el que transcurre su vida cotidiana, se acercan al entorno real de crianza, conocen sus hogares, los lugares de juego y la vida que forma parte del entorno cotidiano de la infancia. La tarea que les espera a las alumnas es incidir en la formación humana en los años donde ocurren los aprendizajes más relevantes de la vida, donde se quiere que los niños y las niñas tenga un mundo justo, equitativo y digno donde vivir (Freire, 2002; Larrosa, 2006).

Como señala Freire (1990), las alumnas ya no estudian solo un repertorio de técnicas de comportamiento, sino que piensan la educación en relación al poder que la constituye, donde el trabajo comunitario, pesado en el primer momento, se transforma en un desafío constante:

«Espero poder pasar los desafios que se presenten día a día, para aceptar a las demás personas como legítimos otros, donde me forme como profesional y sobre todo como una persona capaz de generar relaciones sociales en todo ámbito de mi vida, lo que me facilitará en un futuro llegar a las familias que están detrás de los niños y niñas que pasarán por mi camino, comprendiendo así que no solo trabajo con un niño o niña como individuo, sino teniendo en cuenta que detrás hay todo un núcleo que complementará el aprendizaje que yo le pueda entregar día a día en la jornada diaria».

«Aun teniendo presente que no fue la mejor visita a la comunidad, espero que la próxima sea mejor, no me gusta quedar con la sensación de ir por solo cumplir, ya que, muchas veces llego desmotivada, pero regreso con el corazón lleno y muy feliz, como también con conocimientos nuevos. Mi próximo objetivo es que la próxima semana logre interactuar con más gente, volver de la comunidad con las ganas de regresar nuevamente.»

El rechazo inicial también se transforma a lo largo de los años en el reconocimiento del valor comunitario y la obligatoriedad de un comienzo termina en un compromiso placentero, ético y político, gracias al trabajo permanente y cohesionado con el profesorado entre quienes se construye el sentido de sus 
acciones, que implica educar y educarse con el otro en un proceso de constante alteridad. Los otros se les hacen presentes paulatinamente, como una responsabilidad que ya no pueden eludir. Los otros ya no son unos personajes a los que hay que ir a visitar, sino presencias, rostros que les dicen algo, cuya mirada las traspasa y las interpela a ser generosas, razón por la cual se tornan incapaces de «abordar al Otro con las manos vacías» (Lévinas, 2002, p. 74).

«La percepción de ir a la comunidad por entregar un informe va cambiando, porque ya tú tienes un compromiso y porque te gusta ir, no es porque tienes que entregar una nota.»

«Me costó mucho llegar al real sentido que tenía esta práctica, para mí ahora sí lo tiene.»

«Me dolió saber en realidad cómo era lo que él [Mario] vivía a diario. Desde ayer he pensado mucho en Mario, tratando de idear algún tipo de ayuda que lo llene de esperanza, de que las cosas pueden ser mejor [...] me da impotencia el no saber cómo ayudar...»

Las alumnas han participado, entre otros, con mujeres en grupos y talleres de pintura, poesía, cine arte, yoga, artesanía; con ancianos en la creación de pequeñas huertas familiares; con niños y niñas en la instalación de lugares de juego. Esas experiencias las complementan con los conocimientos disciplinarios en las aulas, tendiendo puentes sobre el vacío epistemológico que existe entre teoría (estudio formal) y práctica (experiencias informales), e identificando el valor formativo de los escenarios informales en la construcción de la profesionalidad docente. El contexto formal de la enseñanza universitaria, generalmente, encuentra dificultades profundas para el logro de aprendizajes con sentido en el proceso de formación profesional, puesto que favorece la formación teórica sobre la práctica, en gran medida porque la segunda es secundaria y meramente repetitiva de ejercicios y, además, muchas veces no tiene consecuencias graves si se hace bien o mal, pues el desempeño queda reflejado en una calificación. La práctica real es aquella en donde hay emergencias, improvisación y autoorganización en medio del desorden propio del devenir cotidiano. Es en el hacer cotidiano, informal, 
donde las alumnas deben resolver situaciones nuevas para la cuales aparentemente no están preparadas, sino que deben buscar soluciones alternativas. Si bien el riesgo de equivocarse es alto, a lo largo de su formación irá disminuyendo significativamente.

La inmersión en la comunidad les permite atribuir significado a sus experiencias, que las analizan y ponderan gracias al estudio teórico. Además, la explicación teórica la reciben desde sus experiencias prácticas y pueden evaluar su utilidad, significado y trascendencia. La participación estudiantil con los miembros de una comunidad se entiende, entonces, desde su sustrato comunitario, lo que implica compartir con sus profesores y profesoras, y compañeras e involucrarse y participar con otros, cuyas circunstancias y emociones surgen de los intereses mutuos de la misma comunidad (Montero, 2007).

«Con las compañeras que participo en estas actividades también se han afianzado los lazos, disfrutamos los días jueves compartiendo con las señoras, pensamos qué de nuevo vamos a aprender y también nos ocupamos de ser una ayuda para ellas, como también, sin duda, he experimentado vivencialmente la influencia que han tenido ellas en mí.»

«Gracias a estos encuentros he podido desarrollar empatía con las vivencias de otras personas, también me mueve el afecto que siento por ellas, disfruto de conversar los días jueves y saber cómo les ha ido en la semana, escuchar sus historias de vida, disfrutar sus risas, pero también conversar desde el amor lo importante que es la resiliencia en la vida».

Los informes que cada mes, durante los cuatro años, entregan las alumnas a sus profesores o profesoras, muestran cómo paulatinamente la experiencia comunitaria influye en su manera de apreciar la vida, los contextos y los aprendizajes formales, ya que se abre la percepción hacia otras realidades antes invisibles. No es fácil desafiar las imposiciones de la realidad hegemónica, como tampoco en las aulas escolares es fácil salir del mundo de la repetición y de las rutinas, porque las escuelas no son culturalmente autónomas y muchas funcionan como meros vehículos de transmisión de habilidades básicas imponiendo modos de conducta y relaciones que se reproducen a sí mismos (Bruner, 1999; Pérez, 2000). Es una manera de interpretar la realidad para que no nos ocasione problemas, razón por la cual tratamos de hacerla 
coherente con nuestros modos de ser, pensar, sentir y actuar; la vamos comprendiendo y nos vamos construyendo al mismo tiempo como personas. Es el mundo cotidiano que se origina en los pensamientos y acciones de los miembros de la sociedad que lo sustentan como real, dando forma, construyendo, negociando e institucionalizando la cultura para reconfortar nuestra vida y nuestros pensamientos (Berger y Luckmann, 1999; Bruner, 1999).

La pedagogía comunitaria facilita el proceso educativo ya que las alumnas estudian de manera no lineal, tensando el orden programático de la clase con la emergencia del devenir contingente, se hacen cargo de colaborar en el mejoramiento cualitativo del desarrollo humano de la comunidad, del cual dan cuenta, como ya se ha señalado, a lo largo de sus cuatro años de formación universitaria. Es un proceso intencional y recíproco que va y viene en un constante bucle de recursividad. Lo que se espera desde el ámbito pedagógico, es que las alumnas desarrollen la capacidad reflexiva y crítica para que generen pautas de acción y movilicen sus propios recursos en favor del fortalecimiento de la comunidad (Montero, 2007). Eso demanda lecturas de las fortalezas más que de las debilidades del entorno y promueve el sentido pedagógico y humano de educar a los párvulos desde la comprensión de los contextos en que estos crecen y se desarrollan. Las alumnas tienen muchas posibilidades de participar genuinamente e involucrarse de manera autónoma en las problemáticas de la comunidad, ya que forman parte de un proceso reflexivo crítico constante y de creación de dinámicas pedagógicas que transforman sus maneras de pensar la educación. Ello favorece los procesos colaborativos de retroalimentación; autoconfianza en sus propias capacidades, conocimientos y habilidades; autonomía y valoración de su práctica; y motivación de logro traducida en el interés cultural permanente (López de Maturana, 2010). Se supone que en ese proceso, el profesorado asume su rol de agentes de cambio y de vicarios de la cultura para que participen con las alumnas en la complejidad del proceso educativo y creen conjuntamente nuevos significados culturales (Bruner, 1999).

Los informes y entrevistas de las alumnas nos muestran la aparición de:

a) Sinergias educativas, donde se relacionan las emergencias espacios temporales con los procesos informales, que implican darse cuenta que la espacio-temporalidad trasciende los muros del aula. Ello puede impactar en 
las estrategias docentes, por ejemplo, la emergencia de preguntas inéditas a partir de las sorpresas y los asombros y el establecimiento de relaciones posibles que le permiten atisbar una mayor complejidad en los procesos de formación:

«Bueno, surgió una instancia muy grata y conmovedora en la exposición [las vecinas exponen el trabajo con las alumnas] ya que todos los presentes nos emocionamos mucho al darnos cuenta que habiamos descubierto esto tan importante para nuestra carrera, y lo más bonito es el lazo que una va creando con las señoras de poder verles cada miércoles y de aprender de lo que más me gusta, que es la unión de equipo tan sincera que ellas tienen, eso me enseñará día a día que, a pesar de que los demás no respondan como a uno le gustaría, se debe continuar y seguir apreciando lo que la vida da.»

b) Reconocimiento de la existencia de la comunidad, de sus costumbres y riqueza de sus miembros, lo que se manifiesta en la percepción de la fuerza educativa del contexto, ya que gracias a las conversaciones con los vecinos van descubriendo emociones, pensamientos, actitudes y capacidades de los miembros de la comunidad, que en otro momento parecían invisibles o insignificantes.

"Entré y saludé a las señoras y mis compañeras aun no llegaban, pude escuchar de lo que hablaban... de la fiesta religiosa a la cual habian asistido ellas el fin de semana, comentaban todo lo que pasó en aquella comunidad religiosa, mientras yo podia preguntarles mis dudas, y pude darme cuenta de la diversidad de creencias que existen, sin que uno las sepa, pero lo más importante de esto es que el ser humano debe respetar y relacionar todo tipo de credo o actuar humano y no atacarse entre ellos, eso nos hará ser una sociedad más aceptable, como dice Maturana "aceptar al otro como legitimo otro".”

«Recuerdo tener la creencia de La Compañía como un sector peligroso, donde uno se bajaba de la micro [bus] y podían asaltarte, estaba con prejuicios y estereotipos que no hacían más que generalizar un sector y a sus habitantes. Es por ello que la práctica en primer año tuvo mucho de probar mis creencias, de conocerlas también ... sin embargo, gracias a esta oportunidad pude también liberarme de muchas creencias que no me pertenecian, más bien eran heredadas, como el miedo y los prejuicios». 
Eso implica la valoración de los otros en un constante bucle de alteridad donde emerge la mirada ética de afirmación mutua, que «no es una mirada depredadora, sino una mirada para el reconocimiento» (Mèlich, 1997), que conduce a la superación de prejuicios y de temores iniciales.

«Muchas veces sin ganas de ir, con mucho cansancio, pero siempre habia algún motivo por el cual asistir, creo que eso era el compromiso que sentíamos con las señoras y que realmente nos fuimos encariñando sin darnos cuenta. Fue una experiencia realmente gratificadora y la cual nunca olvidaré y es de esperar que siempre tengamos un lugar en su corazón para poder recibirnos.»

«Lo conocí como un viejo borracho al que tenía miedo y ahora es don Juan que me espera para conversar.»

c) Construcción de la profesionalidad docente, que se forja a medida que se asienta el compromiso para asistir autónomamente a la comunidad, participar activamente y afectivamente con los vecinos y comprender qué significa ser una educadora situada en el contexto profesional, ético, político y sociocultural. De esa manera se desarrollan las habilidades de liderazgo, cooperación y solidaridad, se amplían los criterios de comprensión del mundo y se afianza la toma de conciencia de la consecuencia de sus acciones.

«Me siento muy feliz de pertenecer a este taller, poco a poco nos fuimos ganando su cariño y confianza [de las mujeres de la comunidad] y creo que tenemos un fuerte lazo de respeto y sobre todo cariño, quién iba a pensar que a partir de la primera visita podríamos conocer ese lugar y personas tan especiales como cada una de las señoras que asisten al taller. Quizás esto es resultado del "lenguajeo", el poder legitimar al otro como legítimo otro, escucharse y poder respetarse dentro de la convivencia, muchas veces sin saber cuál es el final, se generan este tipo de relaciones.»

«Para concluir siento que este proyecto comunitario cada día toma más sentido para mi formación, me doy cuenta que dentro de este contexto tengo muchas cosas que aprender y muchas cosas que entregar, además de darme cuenta y tomar el valor que merece en la historia este sector en particular, generando lazos dentro de la pasión por educar, donde Gabriela Mistral nos da un gran ejemplo de que las cosas se pueden lograr con pasión y perseverancia.» 
A medida que transcurre el proceso de pedagogía comunitaria, las alumnas van estableciendo esos patrones culturales que les permiten comprender e incluirse en la comunidad, y así como la van descubriendo, van reconociendo las sinergias educativas en los espacios y tiempos informales donde se desarrolla la vida comunitaria, generando una recursividad de transformación de los procesos de enseñanza y de aprendizaje universitarios y en su compromiso ético y político en la formación humana.

«La mayoría de las cosas que yo he aprendido en la carrera, como el paradigma que tengo ahora, mi paradigma de pensamiento se basan principalmente en lo que yo he aprendido en base a las prácticas comunitarias, en lo que me han enseñado las personas con las que he trabajado en la comunidad.»

«Valoro el proyecto comunitario en mi formación personal y profesional, cuando voy a mis prácticas docentes sé que las aulas no son espacios exclusivos para aprender y enseñar, sino más bien es el entorno desde sus múltiples escenarios los que aportan y forman a los sujetos como seres sociales».

La carrera de Pedagogía en Educación Parvularia aspira educar a sus alumnas para que vivan «experiencias» que les hagan vibrar, conmover, detener en un tiempo y espacio para recapitular sobre lo hecho y lo por hacer, y que les permita configurar «otras gramáticas y otros esquemas de pensamiento [...] produciendo otros efectos de verdad y otros efectos de sentido» (Larrosa, 2006 , p. 468). Es evidente que queda mucho camino por recorrer, pero al profesorado le asiste la radical novedad de que las alumnas al finalizar su carrera ya no son las mismas, y no por efecto evolutivo ni maduración, sino porque han reconocido y desvelado aquello que se les negó e invisibilizó durante los doce, o más, años de escolarización. El aprendizaje que solo creían obtener entre las cuatro paredes del aula ahora se abre a la inmensidad cultural y al reconocimiento de la vida del otro como legítimo otro en la convivencia (Maturana, 1996). Hay conversaciones, descubrimientos de creencias de la comunidad y contrastación con lo aprendido en el aula. Las alumnas valoran lo aprehendido en la comunidad y lo sienten como el aprendizaje significativo y trascendente que forjó su profesionalidad docente y el sentido de su humanidad.

«Quisiera poder sistematizar más la riqueza de las conversaciones [con los miembros de la comunidad], en ellas he podido conocer las creencias que tienen 
respecto de la mujer, la familia, la sociedad, la política, la niñez, la crianza de los hijos... Es por esto que comprendo el proyecto comunitario de la carrera como una oportunidad de ir más allá de lo aparentemente visible, salir de lo institucional que pone límites a la educación, más bien ha sido formarme desde la deformación que son las creencias preestablecidas que impone la escolarización, disfrazando lo educativo.»

«La formación de educadores requiere de estas prácticas, requiere comprender que no podemos quedarnos ajenos a los contextos donde viven las personas, sus realidades y sus experiencias vitales que los construyen día a día. Los niños y niñas no pueden permanecer encerrados en espacios para ser educados, y nosotras como educadoras de párvulos no podemos trabajar únicamente en estos».

«Hay mucho por hacer, y esta visión se ha desarrollado gracias a esta práctica y a las conversaciones académicas en mi formación. Es por esto que concluyo en la urgencia de ir más allá de lo institucional, de lo necesario que es escuchar lo que tiene que decir la comunidad educativa y desde allí iniciar los cambios y las transformaciones sociales, con el conocimiento que somos una comunidad de aprendizaje donde las relaciones son la clave para una mejor educación, más humana y participativa. ${ }^{6}{ }_{\gg}$

La pedagogía comunitaria abre un territorio de reflexión crítica para superar la escolarización de los procesos educativos, para lo cual el profesorado y alumnado se unen para construir en conjunto una praxis liberadora (Flores, 2014).

\section{Referencias}

Arango, C. (2008). Psicología comunitaria de la convivencia. Cali: Ed. Universidad El Valle.

Berger, P. y Luckmann, T. (1999). La construcción social de la realidad. Buenos Aires: Amorrortu editores.

6. María de los Ángeles Olivares, egresada el año 2005, aportó con la mayor parte de los registros de informes entregados a sus profesores y profesoras cuando era alumna de Pedagogía Comunitaria, y dio su consentimiento para ser identificada. Los otros registros de informes corresponden a sus compañeras del mismo año de egreso, a quiénes se le ha resguardado la identidad. 
BRUNER, J. (1999). La educación, puerta de la cultura. Madrid: Visor.

Calvo, C. (2016). Del mapa escolar al territorio educativo. La Serena: Editorial Universidad de La Serena.

Caron, J. (1996). «Una teoría ecológica para la intervención comunitaria: Acceso y conservación de los recursos». Revista Intervención Psicosocial, Vol. v, $\mathrm{N}^{\mathrm{o}} 14$. Págs. 53-68.

Fals-Borda, O. y Anisur, M. (1991). Acción y conocimiento. Cómo romper el monopolio con investigación-acción participativa. Bogotá: CINEP.

Flores, J. M. (coord.) (2014). Repensar la psicología y lo comunitario en América Latina. Baja California. México: Universidad de Tijuana cut.

Freire, P. (1990). La naturaleza política de la educación. Cultura, poder y liberación. Barcelona: Paidós.

- (2002). Cartas a quien pretende enseñar. México: Siglo XxI.

Larrosa, J. (2006). «Algunas notas sobre la experiencia y sus lenguajes». Estudios Filosóficos, Vol. 55. Nº 160. Págs. 467-480.

LÉvinas, E. (2002). Totalidad e infinito. Ensayo sobre la exterioridad. Salamanca: Sígueme.

López De Maturana, S. (2010). Los buenos profesores. Educadores comprometidos con un proyecto educativo. La Serena: Editorial Universidad de La Serena.

Martín-Baró, I. (2006). «Hacia una psicología de la liberación». Revista Electrónica de Intervención Psicosocial y Psicología Comunitaria, Vol. 1. No 2. Págs. 7-14.

Maturana, H. (1996). El sentido de lo humano. Santiago de Chile: Dolmen.

MèLICH, J.C. (1997). Del extraño al cómplice. La educación en la vida cotidiana. Barcelona: Antrophos.

Montero, M. (2007). Introducción a la psicología comunitaria. Desarrollo, conceptos y procesos. Buenos Aires: Paidós.

Pérez, A. (2000). La cultura escolar en la sociedad neoliberal. Madrid: Morata. 
